\title{
大气扩散集合预报技术和确定性预报技术在日本 福岛核事故全球扩散中的对比分析
}

盛黎 $^{(1)}$, 宋振釒金 ${ }^{(1)}$, 胡江凯 $^{(1)}$, 吕恺 ${ }^{(1)}$, 佟华 ${ }^{(1)}$, 李冰 $^{(2)}$, 乔清党 ${ }^{(2)}$

(1) 国家气象中心数值预报中心, 北京 100081 ;

(2) 环境保护部核与辐射安全中心, 北京 100082

*E-mail: shengli@cma.gov.cn

收稿日期: 2014-01-16; 接受日期: 2014-06-20; 网络版发表日期: 2014-10-21

国家自然科学基金项目(批准号: 41305104)和公益性行业科研专项项目(编号: GYHY201306061)资助

摘要将集合预报技术引入到大气扩散数值模拟中, 这是提高大气扩散数值模拟能力 的一种新的、有效的手段. 2011 年 3 月受日本东北部 9.0 级大地震影响, 日本福岛第一核 电站发生核泄漏事故. 以此次核泄漏事故的全球扩散为研究对象, 通过运用不同的大气扩 散预报技术(集合预报和确定性预报)，模拟了福岛核泄漏释放出来的放射性物质在全球的 扩散过程，并利用监测资料对模拟结果进行了对比分析和评估。文中采用的集合预报技术 共三种, 分别为气象场扰动技术 MET、湍流扰动技术 TUR 和基于大气扩散物理过程参数 化方案的集合预报技术 PHY. 模拟结果显示：集合预报的扩散轨迹模拟技术能更好地模 拟粒子随大气环流扩散的过程; 此外, 与 MET 和 TUR 相比, 确定性预报和集合预报 PHY 模拟扩散范围偏小, 扩散速度偏慢, 且无法模拟出 4 月初日本福岛核放射物质向我国东部 海域扩散的过程. 这是因为 MET 和 TUR 技术核心是对平均速度和湍流速度进行扰动, 这 种三维扰动，会促使污染物向更远方向进行扩散，水平方向扩散连续也更符合监测情况; PHY 主要是对扩散物理过程参数进行扰动, 以垂直速度的扰动为主, 造成扩散范围偏小、 水平方向扩散不连续. 此外, 监测站点与模拟结果的时间序列对比分析结果表明: 模拟结 果均能较好反应浓度趋势演变, 不过模拟浓度偏低, 尤其是确定性预报和 PHY 试验; 此 外, 通过与监测站点的比较得出: 与 PHY 相比, MET 和 TUR 在平均浓度和最大浓度出现 概率上具有更大优势. 本文的研究结果对于丰富大气扩散数值模拟研究, 提高环境应急响 应水平具有参考意义.

集合预报是对未来大气可能状态进行预报模拟 的一种数值模拟方法, 是天气预报由决定论转向随 机论的成果, 代表了当今天气预报发展的重要方法
关键词

集合预报 确定性预报 大气扩散 日本福岛核事故

中文引用格式: 盛黎, 宋振鋪, 胡江凯, 等. 2014. 大气扩散集合预报技术和确定性预报技术在日本福岛核事故全球扩散中的对比分析. 中国科学: 地球科 学, 44: 2554-2564

英文引用格式: Sheng L, Song Z X, Hu J K, et al. 2014. Ensemble Dispersion Modeling VS Deterministic Dispersion Modeling on global dispersion of atmospheric radionuclides from Fukushima Nuclear Accident. Science China: Earth Sciences, doi: 10.1007/s11430-014-4872-x 
(Evan 等, 2000), ECMWF(Molteni 等, 1996), 日本 (Yamaguchi 等, 2009), 中国(田华等, 2007; 任志杰等, 2011)均已建立起各国自己的中期集合预报业务系统. 并且，目前集合预报的应用已拓展到短期天气预报、 月预报乃至季节的气候预测以及水文和大气环境的 预报上来. 有关集合预报的大量实验研究和业务工 作均证明, 集合预报比普通数值预报具有更高的预 报技巧(Buizza 等, 2005; Candille 等, 2007).

与数值天气预报的模拟相似, 大气扩散的预报 模拟同样受到大气流体和扩散过程本身不确定的限 制. Hanna 等(1998)首次将集合预报概念引入到对城 市尺度大气扩散的模拟中来, 从一系列气象初值扰 动成员中选取 50 个分别积分, 以达到对大气光化学 反应的集合预报模拟. Dabberdt 等(2000)对当时大气 扩散集合预报方法的不确定性及其应用等进行了综 述和展望, 在综合各国相关研究的基础上得出: 大气 扩散模拟结果的不确定性主要源自气象强迫场和扩 散模型的选取. 此外, 切尔诺贝利核事故发生后欧洲 开展了大气扩散示踪试验(ETEX-1 和 ETEX-2, European Tracer Experiment; Graziani 等, 1998a, 1998b)和大气扩散模式比较计划的试验(ATMES-1 和 ATMES-2: Atmospheric Transportation Model Evaluation Study; Klug 等, 1992; Albergel 等, 1988). 在此基础上, Galmarini 等(2008)通过收集各国气象驱 动数据和大气扩散模式, 开发了基于不同气象模式 和不同大气扩散模式的超级大气扩散集合预报系统. 不过, 由于此种技术方法维护和计算成本过高, 其应 用受到了很大的限制. Draxler(2002)在前人的基础上 将气象场(主要是风场)扰动技术、湍流扰动技术和扩 散物理参数集合预报技术运用到大气扩散的模拟过 程中, 并集成到 HYSPLIT 模式中来, 并利用美国 ANATEX(Across North America Tracer Experiment)试 验结果对其集合预报的性能进行了验证和评估.

在我国有关大气扩散集合预报技术的研究还不 多见. 其研究主要以中国科学院大气物理研究所 (IAP)王自发等 (2008)的研究为主, 研究以多空气质 量模式为基础, 建立了多模式集合预报模式系统, 通 过对比分析得出集合预报模拟结果与观测值存在显 著相关性，且其效果好于任何单个模式.

本文分别采用三种大气扩散的集合预报技术对 日本福岛核事故全球扩散进行了模拟, 并利用确定 性预报的模拟结果进行了比较分析. 此外, 还通过从
IAEA(International Atomic Energy Agency)和 CTBTO (Comprehensive Nuclear-Test-Ban Treaty Organization) 获取的大量全球辐射监测资料对模拟结果进行验证 和对比分析, 以此检验集合预报技术的模拟性能.

\section{1 试验设计和方法}

\section{1 气象资料}

本文气象驱动场综合采用美国气象环境预报 中心 (NCEP, National Centers for Environmental Prediction) 制作发布的全球再分析资料 (Final Operational Global Analysis data, FNL)数据集和全球 预报场资料(Global Forecast System, GFS)数据集, 空 间分辨率为 $1^{\circ} \times 1^{\circ}$, 时间分辨率为每 $3 \mathrm{~h}$ 一次. FNL 和 GFS 数据集主要为大气扩散模式 HYSPLIT 提供大气 位势高度、温度、风场(水平风场和垂直分量)、相对 湿度、降水以及地面 $2 \mathrm{~m}$ 温度和 $10 \mathrm{~m}$ 风场.

\section{2 大气扩散模式}

大气扩散模式采用美国大气海洋局 (NOAA, National Oceanic and Atmospheric Administration)空 气资源实验室(ARL, Air Resource Laboratory)开发的 HYSPLIT(Hybrid Single-Particle Lagrangian Integrated Trajectory Model)模式(Draxler 和 Hess, 1997, 1998). 该模式基于拉格朗日方法, 是一个集扩散轨迹、浓度 和沉降计算于一体的大气扩散模式系统.

HYSPLIT 模式中, 扩散轨迹的模拟是通过对气 团在 3 维空间的位置积分来完成的. 其计算公式如 下:

$$
\begin{aligned}
P(t+\Delta t)= & P(t)+0.5\left[V(P, \Delta t)+V\left(P^{\prime}, t+\Delta t\right)\right] \Delta t, \\
& P^{\prime}(t+\Delta t)=P(t)+V(P, t) \Delta t,
\end{aligned}
$$

式中, $P$ 表示气团所在的位置, $t$ 表示时间, $\Delta t$ 表示时 间步长, $V$ 风速. $t+\Delta t$ 时刻的气团位置 $P$ 是由气团的初 始位置和气团在 $\Delta t$ 所走位移的矢量和.

对于浓度的模拟, HYSPLIT 支持三种大气扩散 模型: 高斯扩散、粒子随机游走和烟团轨迹扩散模型. 对于水平和垂直方向的污染物扩散, 可以用这三种 模型的进行组合, 不过一般情况下垂直方向不推荐 用高斯扩散模型. 在本文的研究中, 水平扩散采用烟 团 Top-hat 扩散模型, 垂直方向上采用粒子随即游走 模型. 
烟团轨迹扩散模型假设其污染物的释放是一个 个的烟团, 随着时间的演变, 烟团不断变大, 当烟团 大小超过模式格点的分辨率时, 烟团一分为二. 烟团 水平扩散速率可由下面公式可得到,

$$
\mathrm{d} \sigma_{h} / \mathrm{d} t=\left(2 \sigma_{u}\right)^{1 / 2},
$$

由以上公式可知, 烟团的水平扩散速率主要与 湍流速度的标准差有关, 在 HYSPLIT 模式中 $\sigma_{u}$ 的计 算公式如下:

$$
\sigma_{u}=\left(K_{x} / T_{L}\right)^{1 / 2},
$$

式中, $K_{x}$ 是湍流扩散速率, 是高度和大气稳定度的函 数, $T_{L}$ 是拉格朗日时间尺度.

粒子随机游走模型假设污染物以粒子的形态释 放, 跟踪粒子的运动轨迹, 通过计算每个格点内粒子 的数量来确定大气污染物扩散浓度. 粒子的运动轨 迹由以下公式获得

$$
\begin{gathered}
X(t+\Delta t)=X_{\text {mean }}(t+\Delta t)+U^{\prime}(t+\Delta t) \Delta t, \\
U^{\prime}(t+\Delta t)=R(\Delta t) U^{\prime}(t)+v \sigma_{u}\left(1-(R(\Delta t))^{2}\right)^{1 / 2}, \\
R(\Delta t)=\exp \left(-\Delta t / T_{L X}\right),
\end{gathered}
$$

式中, $X$ 表示 $t+\Delta t$ 时刻气团所在的位置, $X_{\text {mean }}(t+\Delta t)$ 表 示气团在 $\Delta t$ 时间内受风场影响所产生的位移, 代表 风场输送的平均速度部分; $U^{\prime}(t+\Delta t)$ 是 $t+\Delta t$ 时刻的水 平脉动速度, 其计算如式(6)所示, 代表湍流速度部 分. 式(6)中, 右边的第一项是相关分量, 第二项是随 机分量或者蒙特卡洛风量. 其中, $R(\Delta t)$ 是拉格朗日自 相关系数, 计算方法如式(7)所示; $v$ 是具有零平均值、 单位标准差的随机函数; $\sigma_{u}$ 是速度脉动标准差, 与所 在高度、混合层厚度、Monin-Obukhov 长度、地表粗 粘度、摩擦速度等有关, 其计算方法如式(8)所示.

\section{3 确定性预报与集合预报技术}

在本文的研究中, 所有大气扩散模拟试验水平 分辨率设定为 $0.5^{\circ} \times 0.5^{\circ}$, 垂直坐标采用地形跟随的 $\sigma$ 坐标系, 一共分成 30 层, 其中第一层距地面 $10 \mathrm{~m}$, 第二层距地面 $75 \mathrm{~m}$, 第三层距地面 $200 \mathrm{~m}$, 扩散模式 顶高约 $26 \mathrm{~km}$ 左右. 为保证大气扩散模拟的稳定性, 时间分辨率为 $6 \mathrm{~min}$.

本文大气扩散集合预报模拟主要通过 HYSPLIT 模式来实现(Draxler, 2002). 文章采用的大气扩散集合 预报技术主要包括: 气象场扰动技术(Meteorological Ensemble, MET)、湍流扰动技术(Turbulence Ensemble, TUR)和扩散物理参数集合预报技术(Physics Parameter

\section{Ensemble, PHY).}

气象场扰动技术其核心主要是通过对气象风场 进行扰动来实现的. 具体的技术方法是在烟团和粒 子扩散过程计算中, 分别在水平和垂直方向添加一 个随机扰动量, 通过在水平方向上 $( \pm 1)$ 个格点 $\left( \pm 0.5^{\circ}\right)$ 的偏移量, 垂直方向上 $( \pm 0.01) \sigma($ 平均约 $( \pm 250) \mathrm{m})$ 的 偏移量，以此构建 27 个集合成员. 值得注意的是，对 气象场格点的扰动并不是在每个时间步长上进行, 而是每隔 $6 \mathrm{~h}$ 扰动一次, 借鉴了 NCEP 繁殖增长模每 $6 \mathrm{~h}$ 更新初值扰动技术有关(Toth 和 Kalnay, 1993). 气 象扰动场技术集合成员如表 1 所示.

湍流扰动集合预报技术是通过在湍流速度脉动 标准差的计算中引入 27 个随机扰动因子(确定性预报

\begin{tabular}{|c|c|c|c|}
\hline 集合成 & $\Delta X$ & $\Delta Y$ & $\Delta Z$ \\
\hline 1 & 0 & 0 & -1 \\
\hline 2 & 0 & 1 & -1 \\
\hline 3 & 0 & -1 & -1 \\
\hline 4 & 1 & 0 & -1 \\
\hline 5 & 1 & 1 & -1 \\
\hline 6 & 1 & -1 & -1 \\
\hline 7 & -1 & 0 & -1 \\
\hline 8 & -1 & 1 & -1 \\
\hline 9 & -1 & -1 & -1 \\
\hline 10 & 0 & 0 & 0 \\
\hline 11 & 0 & 1 & 0 \\
\hline 12 & 0 & -1 & 0 \\
\hline 13 & 1 & 0 & 0 \\
\hline 14 & 1 & 1 & 0 \\
\hline 15 & 1 & -1 & 0 \\
\hline 16 & -1 & 0 & 0 \\
\hline 17 & -1 & 1 & 0 \\
\hline 18 & -1 & -1 & 0 \\
\hline 19 & 0 & 0 & 1 \\
\hline 20 & 0 & 1 & 1 \\
\hline 21 & 0 & -1 & 1 \\
\hline 22 & 1 & 0 & 1 \\
\hline 23 & 1 & 1 & 1 \\
\hline 24 & 1 & -1 & 1 \\
\hline 25 & -1 & 0 & 1 \\
\hline 26 & -1 & 1 & 1 \\
\hline 27 & -1 & -1 & 1 \\
\hline
\end{tabular}
中只产生一个随机数)来实现的(Challa 等, 2008). 式 (8)分别显示的是确定性预报和湍流扰动集合预报技 术中对于湍流脉动速度的计算方法.

\section{表 1 气象场扰动技术集合成员水平和垂直扰动量}




$$
\begin{cases}v \sigma_{u}, & \text { 确定性预报, } \\ v(I S E E D)_{27} \sigma_{u}, & \text { 湍流扰动的集合预报, }\end{cases}
$$

式中, ISEED 是随机数, 在本文研究中共 27 个. 对于 确定性预报, 随机分量是由一个随机数产生, 而湍流 扰动的集合预报是由 27 个随机数扰动而成. 理论而 言，三维粒子扩散模型只有当释放的粒子足够多的 时候，其模拟结果才能与真实情况近似; 但是在实际 模拟过程中由于受到计算条件和计算机时的限制往 往采用有限的粒子个数. 通过对随机分量进行扰动, 不仅可以大大地减少计算量, 同时又能保证模拟结 果接近污染物扩散的真实情况. 此外, 本文的研究中 针对日本福岛核事故的全球扩散模拟，模拟时间长 达一个多月，计算量巨大，因此构建一个基于湍流扰 动的集合预报技术尤为必要.

基于大气扩散物理过程参数化方案集合预报技 术是通过采用不同大气扩散模型、混合层高度计算方 法、水平和垂直的拉格朗日时间尺度计算方法等构建 不同的集合成员来实现的. 在本文的研究中, 通过 20 个不同扩散物理过程参数化方案得到 20 个集合成 员, 如表 2 所示.

\section{表 2 基于大气扩散物理参数集合预报技术采用的 20 个集合成员}

\begin{tabular}{|c|c|}
\hline 集合成员 & 大气扩散物理参数设定 \\
\hline 1 & 水平和垂直均采用 3 维粒子扩散模型 \\
\hline 2 & 水平方向采用高斯扩散, 垂直采用 3 维粒子扩散模型 \\
\hline 3 & $\begin{array}{l}\text { 水平采用分裂的烟团扩散模型，垂直采用 } 3 \text { 维粒子 } \\
\text { 扩散模型 }\end{array}$ \\
\hline 4 & 采用经验的非线性烟团扩散速率 \\
\hline 5 & 采用温度廓线的方法计算混合层高度 \\
\hline 6 & 采用 TKE 方法计算混合层高度 \\
\hline 7 & 混合层高度设定为 500 m \\
\hline 8 & 混合层高度设定为 $1000 \mathrm{~m}$ \\
\hline 9 & 混合层高度设定为 $1500 \mathrm{~m}$ \\
\hline 10 & 设定最小的混合层高度为 $125 \mathrm{~m}$ \\
\hline 11 & 垂直混合强度采用混合层内平均值 \\
\hline 12 & 通过流场来计算水平湍流 \\
\hline 13 & 采用风/温廓线来计算边界层稳定度 \\
\hline 14 & 采用 Beljaars 的湍流参数化方案 \\
\hline 15 & 采用 TKE 的湍流参数化方案 \\
\hline 16 & 垂直湍流的计算采用: TKER $=0.36 * w^{\prime 2}\left(u^{\prime 2}+v^{\prime 2}\right)^{-2}$ \\
\hline 17 & 垂直的拉格朗日时间尺度设定为 $200 \mathrm{~s}$ \\
\hline 18 & 水平拉格朗日时间尺度设定为 $5400 \mathrm{~s}$ \\
\hline 19 & 垂直的拉格朗日时间尺度设定为 $400 \mathrm{~s}$ \\
\hline 20 & 水平拉格朗日时间尺度为 $21600 \mathrm{~s}$ \\
\hline
\end{tabular}

\section{4 福岛核事故排放源和监测数据}

对福岛核事故放射性物质释放源项的评估，如泄 漏种类、总量大小、泄漏速率和高度特征等, 是核事故 全球扩散模拟的重要内容. Chino 等(2011) 采用逆向评 估办法, 在大量监测资料的基础上, 利用 WSPEEDIII(the Worldwide version II of System for Prediction of Environmental Emergency Dose Information)的逆向模 拟结果对 3 月 14 日至 4 月 5 日间放射性物质释放源项 特征进行了评估. Katata 等(2012a，2012b)利用离福岛 核电站稍远的监测资料，基于 WSPEEDI-II 的正向大 气扩散模拟结果，不仅对早期的源项特征进行了评估， 还将评估结果延长到了 5 月 1 日. Terada 等(2012)在综 合各类评估数据的基础上，综合利用中尺度的大气扩 散模拟结果和获得的地表空气浓度、地表沉积量等监 测资料基础上对原排放源数据进行了验证和修正，文 章采用 Terada 等(2012)的评估结果作为大气扩散模拟 试验的排放源数据.

本文中所用到的我国境内大气辐射监测数据来 自于我国环境保护部(国家核安全局)在日本福岛核 事故期间公布的我国主要城市环境辐射水平监测数 据. 对于境外站点的辐射监测数据来自于 IAEA 收集 到的全球各国在日本福岛核事故期间的监测结果和 CTBTO IMS(International Monitoring System)40 多个 站点的监测数据.

\section{2 结果分析}

\section{1 福岛核事故扩散轨迹模拟分析}

针对日本福岛核事故的全球扩散轨迹模拟，本 文利用 HYPSLIT 模式分别设计两组轨迹模拟试验: 基于确定性预报的控制试验(CTL_TRAJ)和基于气象 场扰动技术集合预报试验(ENS_TRAJ). 两组试验排 放高度均设定在 $3000 \mathrm{~m}$, 一般情况下这一高度是边 界层顶所在的高度，代表福岛核事故核放射物质在 爆炸或边界层夹卷至边界层顶以后的扩散轨迹, 能 表示扩散最快那部分粒子的运动状态，方便与 CTBTO IMS 站点首次监测时间进行比较. 模拟时间 2011 年 3 月 12 日 00 时至 4 月 20 日 00 时(世界时). 模 拟结果与 CTBTO IMS 站点首次监测到放射性物质的 时间对比如图 1 所示. CTBTO 观测结果显示: 3 月 12 日日本 Takasaki 监测站(JPP38)首次监测到了来自 


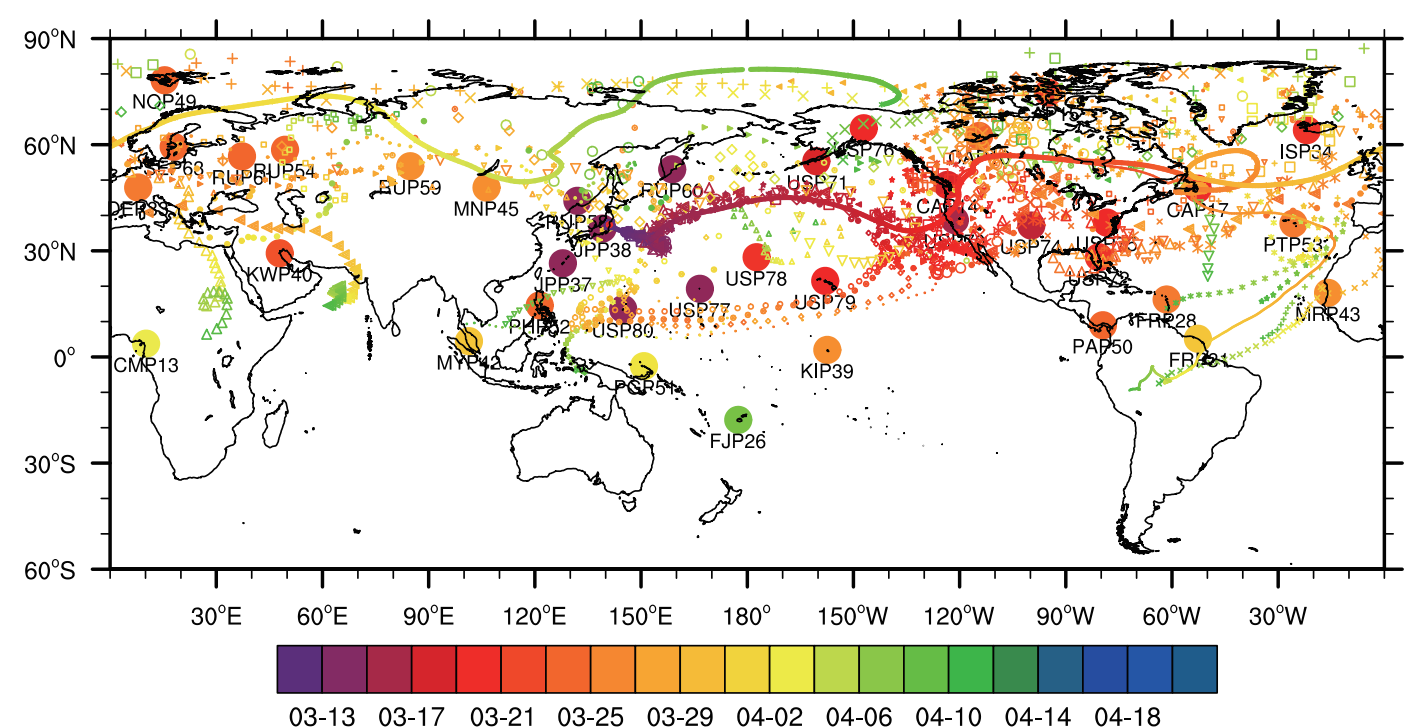

图 1 HYSPLIT 模拟 CTL_TRAJ, ENS_TRAJ 扩散轨迹及与 CTBTO IMS 首次监测时间分布示意图(世界时)

细实线: CTL_TRAJ; 粗实线: ENS_TRAJ 的集合平均; 大圆点: CTBTO IMS 首次监测站点; 带标记线条: 轨迹集合预报的 27 个成员

$200 \mathrm{~km}$ 以外的福岛核事故释放出来的放射性物质; 14 日俄罗斯远东地区的两个监测站点 (RUP58 和 RUP60)监测到了 I-131；4，5 天以后核辐射烟团抵达 北美西海岸(CAP14 和 USP74); 22 日冰岛 Rehkjavik (ISP34)检测到来自福岛的放射性物质; 随后, 欧洲 各国均相继监测到; 至 3 月 28 日蒙古也已监测到从 上游扩散而来的日本福岛放射性物质，这表明至此 放射性物质的扩散已弥散至整个北半球. 进入 4 月份 后辐射烟团继续扩散, 4 月 3 日南半球 FJP 站点已监 测到日本福岛核事故泄漏出来的放射性物质. 至 2011 年 5 月底, CTBTO 40 多个辐射监测站点均已监 测到来自日本福岛核事故泄漏出来的放射性物质。

根据图 1 的模拟结果显示: 模拟前期(3 月 12 日 至 3 月 24 日), CTL_TRAJ 与 ENS_TRAJ 轨迹与 CTBTO 首次监测站点时间基本一致, 且各集合成员 间也具有较高的一致性; 模拟后期(3 月 24 日以后), CTL_TRAJ 模拟轨迹在北非西海岸受大西洋东风气 流影响折向南扩散, 并终止于南美东北部地区, ENS_TRAJ 模拟轨迹集合平均则主要随北半球中高 纬度高空西风气流影响持续扩散, 在 3 月底完成其扰 地球一周的扩散, 其结果与监测结果基本一致. 此外, 各集合成员在全球的扩散显示: 大部分集合成员在 北半球中高纬扩散, 随着积分时间的延长成员逐渐 发散. 综合而言, 与确定性预报模拟结果相比, 集合 预报技术模拟结果与 CTBTO 监测分布更为一致; 而
且, 集合预报模拟结果也能为放射性物质在全球的 扩散提供概率性预报产品.

\section{2 福岛核事故全球扩散数值模拟分析}

基于确定性和集合预报技术，本文利用 HYSPLIT 模式对日本福岛核事故的全球扩散进行了 数值模拟. 数值模拟试验共分为 4 组: (1) 基于确定 性预报的控制试验(CTL); (2) 基于气象场扰动技术 的集合预报试验(MET); (3) 基于湍流扰动技术的集 合预报试验(TUR);（4）基于扩散过程物理参数的集 合预报试验(PHY). 模拟中泄漏源项的数据采用 Terada 等(2012)的评估源项数据.

本文对福岛核事故全球扩散的分析以放射性物 质全球扩散对人体的危害为出发点. 核放射物质对 人体的危害主要通过两种途径来实现：辐射外照射 和辐射内照射. 辐射外照射主要是地面(或水面)累积 沉降等对人体外部造成辐射危害; 辐射内照射主要 通过空气吸入(与地表空气浓度紧密相关)、食品食入 等途径沉积在体内，从而形成持续性的内照射危害. 本文将从地面累积沉降和地表空气浓度两方面来对 比分析确定性预报和集合预报大气扩散模拟结果.

\subsection{1 地表累积沉降}

福岛核事故期间北半球的地面风场示意图见网 络版附图 1 (http:earth.scichina.com), 确定性预报和集 
合预报四组试验地面累积沉降水平分布图见网络版 附图 2, 综合附图 1 和图 2 的结果可知:

3 月 12 15 日期间, 东北亚地区以偏西气流为主, 福岛核事故释放出来放射性物质向福岛东北方向扩 散, 主要影响日本福岛附近及其东北方向的西北太 平洋海域. 这一阶段的扩散模拟结果显示: CTL 与 MET、TUR 和 PHY 试验模拟的扩散趋势一致, CTL 扩散范围偏小且浓度值偏低.

3 月 16 19 日, 日本附近地区主要受高压系统影 响, 偏西气流携带放射性物质向下游方向扩散, 历时 7 天含放射性物质烟团抵达美国西海岸附近(CTBTO 的监测结果显示 3 月 18 日美国加利福利亚监测到来 自日本的放射性物质). 此阶段的模拟显示：各试验 模拟扩散范围基本一致, 不过 CTL 扩散速度明显滞 后于 MET, TUR 和 PHY; 较确定性预报 CTL, 集合预 报试验 MET, TUR 和 PHY 的模拟结果与 CTBTO 监 测结果具有更高的一致性. 此外，不同的集合预报技 术模拟结果也各不一样, MET 与 TUR 的模拟结果基 本一致, PHY 模拟的扩散范围偏小.

3 月20 25日，东北亚地区受低压系统东移影响， 近地层偏北气隽带放射性物质向偏南方向扩散, 新 释放的放射性物质主要影响福岛以南的日本地区, 同时 21 23 日日本大部分地区的降水有利于放射性 物质的湿沉降. 此外, 太平洋北部和北美以持续的偏 西气流为主, 受此影响早期释放的放射性物质持续 向下游方向扩散, 辐射烟云抵达欧洲地区(监测结果 显示: 3 月 23 日左右欧洲各国相继监测到放射性物 质). 这一阶段的模拟: CTL 和 PHY 模拟结果相似, 扩散前沿抵达美国西海岸或美国西部地区，大气扩 散的速度明显滞后于 MET, TUR 以及 CTBTO 监测结 果; 集合预报试验 MET 和 TUR 模拟结果显示扩散前 沿抵达大西洋和北欧地区, 稍滞后于监测结果. 不同 的集合预报扰动技术, MET 与 TUR 模拟结果具有较 高一致性, PHY 则显著滞后于 MET 和 TUR 的扩散.

3 月 26 30 日, 日本地区受高空槽减弱东移的影 响, 西北气流携带放射性物质向下游方向扩散; 3 月 31 日至 4 月 7 日, 随着高压系统的自西向东移动, 日 本以南至我国东部洋面出现持续的偏东气流, 受此 影响放射性物质向西扩散, 并影响我国(3 月 25 日我 国在黑龙江省首次监测到来自日本的放射性物质, 4 月 7 日左右我国大部分城市监测到的 I-131 的浓度峰 值, 盛黎等, 2013). 与此同时, 早期释放的放射性物
质持续向下游方向, 经欧洲上空抵达中亚地区. 这一 阶段的模拟结果显示：集合预报试验 MET 和 TUR 较 好地模拟出了放射性物质从日本向我国东部海域扩 散的过程, CTL 和 MET 则没有. 此外, 四组试验模拟 结果均显示截至 4 月 7 日辐射烟云前沿经欧洲地区, 抵达中亚; 不过, CTL 和 PHY 模拟扩散范围较 MET 和 TUR 小. 4 月 7 日至 4 月 15 日, 日本中南部地区受 高压系统影响, 西南气流携带福岛核事故新释放的 放射性物质.

向高纬地区扩散, 影响俄罗斯远东沿海地区和 太平洋北部海域. 在此阶段, CTL, MET, TUR 和 PHY 试验对于放射性物质向高纬地区的扩散的模拟结果 基本一致. 对于中亚地区的扩散模拟, MET 和 TUR 结果比较一致, 至 4 月 15 日放射性物质覆盖北半球 大部分区域; CTL 和 PHY 试验扩散范围较前期没有 显著变化, 与 4 月 6 日扩散范围基本相当.

综合 4 组试验对日本福岛核事故全球扩散模拟 结果, 可得出以下结论: 4 组试验扩散趋势基本一致, CTL 和 PHY 模拟扩散范围较 MET 和 TUR 偏小, 尤 其是在扩散后期, CTL 和 PHY 难以模拟出放射性物 质在整个北半球的扩散过程. 此外, TUR 和 PHY 试验 还能较好地模拟出福岛放射性物质向我国东部海域 的扩散过程, CTL 和 MET 则不能. 由模拟结果可知, MET 和 TUR 中扩散到超远距离的放射性物质较多; 而 CTL, PHY 扩散出去的较少, 留在附近地区的偏多. 这可能与集合预报的扰动方法有关, MET 和 TUR 扰 动技术, 其最终的核心内容是对扩散的风场进行扰 动, MET 是对平均风速进行扰动, TUR 是对湍流速度 进行扰动, 包括水平风速的扰动和垂直风速的扰动, 这种风场扰动(尤其是水平风场扰动)利于放射性物 质扩散到更远的地方; 而 PHY 核心内容是对扩散过 程中的各项扩散参数, 如混合层厚度、拉格朗日时间 尺度等进行扰动, 以对垂直风场的扰动为主, 所以不 利于放射性物质的水平输送.

\subsection{2 地表空气浓度数值模拟分析}

为进一步考察大气扩散确定性预报与集合预报 模拟能力, 本文对 CTL, MET, TUR 和 PHY 试验模拟 的地表空气浓度最大值及最大浓度出现时间进行了 比较分析, 如图 2 所示. 地表空气浓度最大值的模拟, 能反映不同的预报技术对于极大值的模拟能力; 极 大浓度出现时间的模拟, 则能反映模式对大气扩散 

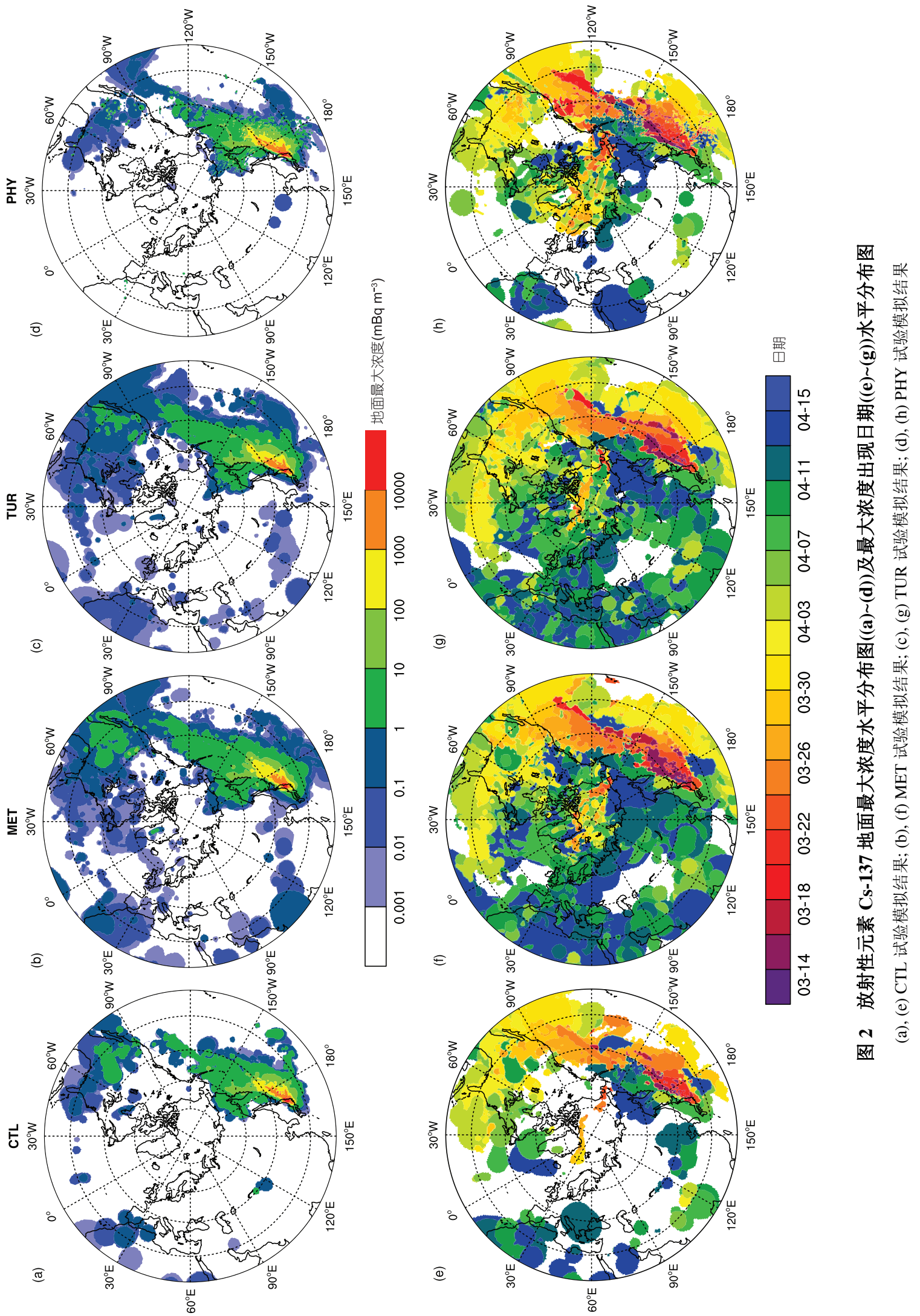
速度的把握, 偏快还是偏慢. 4 组试验最大浓度水平 分布(图 3(a) (d)) 显示: 日本福岛核事故放射性物质 的地面最大浓度区域 $\left(>100 \mathrm{mBq} \mathrm{m}^{-3}\right)$ 主要发生在日本 及其以东的太平洋地区; 次大浓度区主要位于北美 地区, 最大浓度值大约在 $10 \mathrm{mBq} \mathrm{m}^{-3}$ 左右. 不过, 4 组试验结果也存在不同之处: CTL 和 PHY 试验模拟 结果的高浓度范围较小, 主要集中在日本附近及其 下风向的北太平洋地区, 远程扩散比较少; MET 和 TUR 模拟的高浓度范围较大, 试验模拟到污染物在 欧洲和亚洲的缓慢扩散过程. 模拟结果的不同与这 三种集合预报技术的扰动方法有关, MET 和 TUR 对 三维风场(尤其是水平风场扰动)的扰动技术, 更利于 污染物的水平扩散, 而 PHY 中以垂直风速为主的扰
动技术, 则不利于污染物的水平输送.

最大浓度的出现日期如图 3(e) (h)所示, 四组试 验模拟结果均显示: 日本及其下游的西北太平洋地 区最大浓度出现在 3 月 17 日左右; 北美地区浓度极 大值出现在 3 月底; 中亚地区(包括我国西北地区)在 4 月初出现地面浓度的极大值. 不过四组试验模拟结 果也存在一些不同之处: 集合预报试验 MET, TUR 和 PHY 模拟的大气扩散速度比确定性预报 CTL 快 3 5 天左右, 与观测更为一致; 集合预报试验模拟到 了污染物向极地的扩散过程, 确定性预报则没能; 此 外, CTL 和 PHY 试验模拟的污染物的远程扩散过程 存在不连续性. 模拟结果从侧面反映出, MET和 TUR 的扩散几乎是整个北半球、连续的扩散, CTL 和 PHY
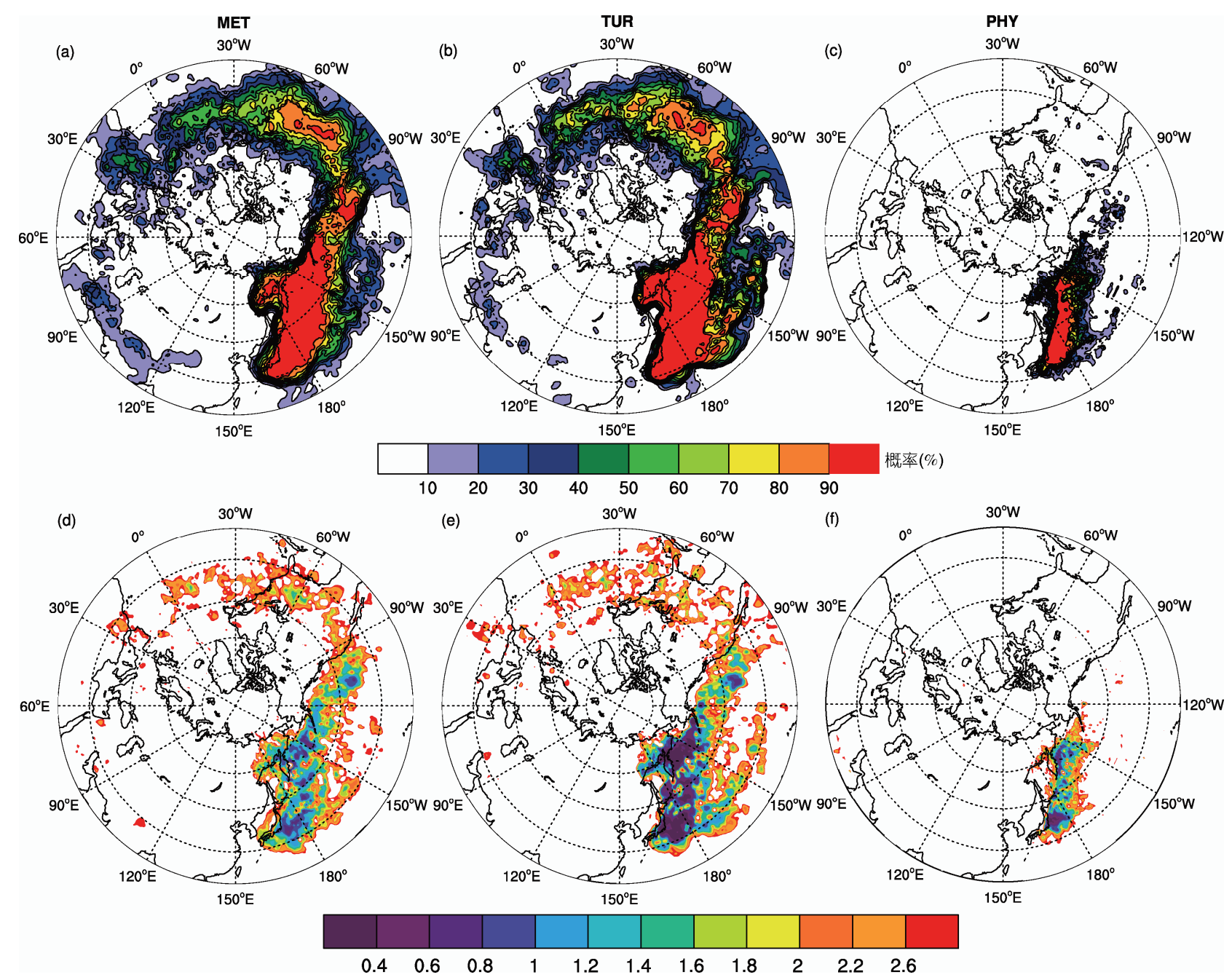

图 3 放射性元素 Cs-137 地面最大浓度大于 $1.0 \mathrm{mBq} \mathrm{m}^{-3}$ 概率 $((\mathbf{a}) \sim(\mathrm{c}))$ 及归一化集合离散度 $((\mathbf{d}) \sim(\mathbf{f}))$ 的水平分布图

(a), (d) MET 试验模拟结果; (b), (e) TUR 试验模拟结果; (c), (f) PHY 试验模拟结果 
则存在不连续性; MET 和 TUR 更符合 CTBTO 的监 测结果和真实的大气扩散状态 (CTBTO 在北半球所 有的监测站点均监测到了放射性物质).

\section{3 不同集合预报技术的模拟结果比较分析}

与确定性的大气扩散预报技术相比, 大气扩散 集合预报技术能提供更多的概率预报信息. 为了比 较不同的大气扩散集合预报技术在概率预报产品上 的差异, 本文就 MET, TUR 和 PHY 三种试验模拟的 地表最大浓度大于 $1.0 \mathrm{mBq} \mathrm{m}^{-3}$ 概率预报和归一化离 散度水平分布进行了比较分析，其结果如图 3 所示. 结果显示: MET 和 TUR 模拟地表最大浓度大于 1.0 $\mathrm{mBq} \mathrm{m}^{-3}$ 大概率范围要远大于 PHY. 日本福岛核事故 期间美国 IMS 的 10 个监测站点显示(Biegalski 等, 2011), 所有站点 I-131 最大浓度均大于 $1.0 \mathrm{mBq} \mathrm{m}^{-3}$, 6 个站点 Cs-137 浓度大于 $1.0 \mathrm{mBq} \mathrm{m}^{-3}$. 这表明, MET 和 TUR 模拟结果与监测数据更趋一致.

集合预报产品的另一种重要表现形式是离散度, 由于福岛核事故期间全球的浓度分布差异较大 $\left(10^{-6} \sim 10^{1}\right)$, 因此采用归一化的离散度 NS. MET, TUR 和 PHY 试验中地表最大浓度归一化的离散度分布如 图 3(d) (f)所示. 三组试验 NS 数值随着扩散距离而 变大, NS 水平与其对应的概率分布相似; 值得注意 的是, 在日本福岛附近和西北太平洋地区, TUR 试验 对应 NS 的低值区 $(\mathrm{NS}<0.6)$, 而 MET 和 PHY 的 NS 值偏大 $(>0.8)$. 这表明: 对于中近距离的扩散 TUR 的 离散较小, MET 和 PHY 相当; 这可能与 TUR 试验中 湍流速度扰动量偏小 (相对于全风速)有关; 对于 MET 试验由于核心是对平均风速进行扰动, 其扰动 量可能较大, 造成各集合成员地表空气浓度差异显 著; 而 PHY 试验虽然对水平风速的影响不大, 但是 不同的扩散模型、混合层高度等的计算对边界层放射 性物质浓度分布具有非常重要的意义, 地表空气浓 度的差异亦比较显著. 当扩散较远时(超远距离扩散), MET, TUR 和 PHY 的离散度分布基本一致, 这可能 是因为当扩散达到一定距离, 由于误差或者集合成 员模拟差异的累积不同成员之间差别越大越大, 造 成离散度偏高.

\section{4 模拟结果与监测结果的比较分析}

为了对福岛核事故大气扩散确定性预报和集合 预报模拟结果进行验证, 本文选取来自北半球八个
国家的 16 个监测站点来对模拟结果进行对比分析, 其结果如网络版附图 3 所示. 由附图 3 可知: 亚洲区 域, 中国和韩国监测到的 Cs-137 地面最大浓度大约 在 $1.0 \mathrm{mBq} \mathrm{m}^{-3}$, 模拟结果整体较监测值偏小 $1 \sim 2$ 个量 级左右. 长春、北京、上海、武汉以及韩国的 Gunsan 均呈现先低后高的趋势变化, 峰值大约出现在 4 月 7 日左右，这与 4 月 2 日我国东部海域出现的持续偏东 风有关; 广州由于受热带气候影响其监测浓度呈双 峰型的变化. 值得注意的是, 集合预报与确定性预报 模拟结果基本一致, 这可能与我国距离日本距离较 近有关.

对于北美站点，与亚洲地区的先低后高的趋势 演变不同, Las Vegas 和 Oahu 监测站点呈现先高后低 的变化趋势, 监测浓度范围约在 $0.1 \sim 10 \mathrm{mBq} \mathrm{m}{ }^{-2}$, 模 拟结果与监测趋势基本一致, Las Vegas 模拟结果偏 小, Oahu 偏大, 模拟误差约在 $( \pm 1)$ 个量级左右. 四组 试验与观测结果比较来看, CTL 和 PHY 模拟浓度较 MET 和 TUR 偏低.

欧洲地区地表空气浓度呈单峰型的变化, 高浓 度值出现在 4 月初. 4 组试验模拟结果模拟均模拟出 了地表空气浓度单峰型的变化, 不过模拟浓度偏低, 尤其是控制试验 CTL 和集合预报试验 PHY.

综合而言, 四组试验均能较好模拟出各站点地 表空气浓度的时间演变趋势, 整体而言模拟浓度偏 低, 尤其是 CTL 和 PHY. 不过，由于扩散模式分辨 率、插值误差以及监测资料的代表性等原因，与确定 性预报相比较，集合预报模拟在时间序列上对于趋 势的把握方面优势并不显著. 对于不同的集合预报 技术，综合监测浓度(网络版附图 3(b) (d)) 和集合预 报概率产品(图 3(a) (d)) 可知：各监测站点最大监测 浓度基本均在 $1 \mathrm{mBq} \mathrm{m}^{-3}$ 左右, 除东亚地区外, 其他 监测站点最大浓度分布均落在 MET 和 TUR 概率分 布 $>70 \%$ 的范围之内，但落在 PHY 概率分布之外. 由 此可知, 与 PHY 相比, MET 和 TUR 对于平均浓度和 对最大浓度出现概率的模拟具有更大的优势.

\section{3 结论}

本文综合利用美国 NCAR/NCEP FNL 再分析资 料和 GFS 预报场作为气象驱动场, 运用 HYSPLIT 模 式，针对日本福岛核事故全球扩散开展了确定性预 报和集合预报的数值模拟试验及其对比分析研究. 通 
过各国环保部监测资料、IAEA 和 CTBTO IMS 监测资 料与试验模拟结果的对比分析, 得到的结论如下.

(1) 对于长时间、远距离扩散模拟, 在扩散轨迹 方面, 集合预报技术较确定性预报技术对具有更好 的模拟性能. 确定性预报的模拟由于在长时间的积 分过程中误差不断累积, 可能轨迹偏离大气环流的 平均状态, 从而导致轨迹的终止或消失. 相反, 集合 预报由于在积分过程中通过引入误差扰动量, 通过 对多个成员分别积分, 能最大限度地模拟出污染物 在大气中可能的扩散状态.

(2) 确定性预报与集合预报扩散趋势和最大浓 度的模拟基本一致; CTL 和 PHY 扩散范围偏小, 扩散 速度偏慢, 难以模拟出放射性物质在整个北半球的 持续扩散过程. 此外, 集合预报 TUR 和 PHY 试验能 较好地模拟出福岛放射性物质向我国东部海域的扩 散过程, CTL 和 MET 则不能. 这可能与不同集合预报 试验采用的扰动方法有关, MET 和 TUR 其核心内容 分别是对平均速度和湍流速度进行扰动, 三维的风 速扰动(尤其是水平扰动)促使污染物向更远方向进 行扩散, 水平扩散更连续也更符合实际监测情况;
PHY 主要是对扩散物理过程参数进行扰动, 其核心 是对垂直速度进行扰动, 造成放射性物质很难扩散 到超远距离以及扩散水平方向不连续.

(3) 通过对不同的集合预报技术概率产品和离 散度模拟结果表明: MET 和 TUR 在大于 $1.0 \mathrm{mBq} \mathrm{m}^{-3}$ 的概率分布基本一致, 更符合监测结果; PHY 中高浓 度的大概率主要分布在福岛下游较小范围的西北太 平洋地区. 对于中近距离的扩散 TUR 的离散较小, MET 和 PHY 离散度相当; 这可能与不同的集合预报 方法扰动量大小、扩散物理过程参数对扩散过程的影 响等因素有关.

(4) 通过浓度模拟结果与亚洲、美洲和欧洲共 16 个监测站点的对比分析可知, 模拟结果在浓度整体 趋势方面具有一定的模拟能力, 不过模拟浓度偏低, 尤其是 CTL 和 PHY 试验. 此外, 由于扩散模式分辨 率、插值误差以及监测资料的代表性等, 与确定性预 报相比较, 集合预报模拟在时间序列上对于趋势的 把握方面优势并不显著. 对于不同的集合预报技术, 与 PHY 相比, MET 和 TUR 对于平均浓度和对最大 浓度出现概率的模拟具有更大优势. 室的 Draxler 博士，他对 HYSPLIT 的模拟提供了许许多多的技术支撑和帮助。感谢两位匿名评审人对文章提 出的许多宝贵意见.

\section{参考文献}

任志杰，陈静，田华. 2011. T213 全球集合预报系统物理过程随机扰动方法研究. 气象, 37: 1049-1059

盛黎，周斌，孙明华，等. 2013. 日本福岛核事故对我国辐射环境影响的监测与分析. 气象, 39: 1529-1538

田华, 邓国, 胡江凯. 2007. 全球 T213 数值集合预报业务系统简介. 见: 中国气象学会 2007 年年会天气预报预警和影响评估技术分会场 论文集

王自发, 庞成明, 朱江, 等. 2008. 大气环境数值模拟研究新进展. 大气科学, 32: 987-995

Albergel A D, Martin B, Strauss B, et al. 1988. The Chernobyl accident: Modeling of dispersion over Europe of the radioactive plume and comparison with air activity measurements. Atmos Environ, 22: 2431-2444

Biegalski S R, Bowyer T W, Eslinger P W, et al. 2011. Analysis of data from sensitive U.S. monitoring stations for the Fukushima Dai-ichi nuclear reactor accident. J Environ Radioact, 114: 15-21

Buizza R, Houtekamer P L, Toth Z, et al. 2005. A comparison of the ECMWF, MSC, and NCEP global ensemble prediction system. Mon Weather Rev, 133: 1076-1097

Candille G, Cote C, Houtekamer P L, et al. 2007. Verification of an ensemble prediction system against observations. Mon Weather Rev, 135: 2688-2699

Challa V S, Indrcanti J, Baham J M, et al. 2008. Sensitivity of atmospheric dispersion simulations by HYSPLIT to the meteorological predictions from a meso-scale model. Environ Fluid Mech, 8: 367-387

Chino M, Nakayama H, Nagai H, et al. 2011. Preliminary estimation of release amounts of ${ }^{131} \mathrm{I}$ and ${ }^{137} \mathrm{Cs}$ accidentally discharged from the Fukushima Daiichi Nuclear Power Plant into atmosphere. J Nuclear Sci Tech, 48: 1129-1134 
Dabberdt W F, Miller E. 2000. Uncertainty, ensembles and air quality dispersion modeling: Applications and challenges. Atmos Environ, 34 : $4667-4673$

Draxler R R. 2002. Verification of an ensemble dispersion calculation. J Appl Meteorol, 42: 308-317

Draxler R R, Hess G D. 1997. Description of the HYSPLIT_4 modeling system. NOAA Tech. Memo. ERL ARL-224, NOAA Air Resources Laboratory, Silver Spring, MD. 24

Draxler R R, Hess G D. 1998. An overview of the HYSPLIT_4 modeling system of trajectories, dispersion, and deposition. Aust Meteor Mag, 47 : 295-308

Epstein E S. 1969. Stochastic dynamic prediction. Tellus, 21: 939-759

Evans R E, Harrison M S J, Graham R J, et al. 2000. Joint medium-range ensemble from the Met. Office and ECMWF systems. Mon Weather Rev, 128: 3104-3127

Galmarini S, Bianconi R, Bellasio, et al. 2008. Forecasting the consequences of accidental releases of radionuclides in the atmosphere from ensemble dispersion modelling. J Environ Radioact, 57: 203-219

Graziani G, Mosca S, Klug W, 1998a. Real-time long-range dispersion model evaluation of ETEX first release. EUR17754/EN. Office for Official Publications of the European Commission, Luxembourg

Graziani G, Klug W, Galmarini G, et al. 1998b. Realtime long-range dispersion model evaluation of ETEX second release. EUR 17755/EN. Office for Official Publications of the European Commission, Luxembourg

Hanna S, Chang J C, Fernau M E. 1998. Monte Carlo estimates of uncertainties in predictions by a photochemical grid model (UAM-IV) due to uncertainties in put variables. Atmos Environ, 32: 3619-3628

Katata G, Ota M, Terada H, et al. 2012a. Atmospheric discharge and dispersion of radionuclides during the Fukushima Dai-ichi Nuclear Power Plant accident. Part I: Source term estimation and local-scale atmospheric dispersion in early phase of the accident. J Environ Radioact, 109: 103-113

Katata G, Terada H, Nagai H, et al. 2012b. Numerical reconstruction of high dose rate zones due to the Fukushima Dai-ichi Nuclear Power Plant accident. J Environ Radioact, 111: 2-12

Leith C E. 1974. Theoretical skill of Monte Carlo forecasts. Mon Weather Rev, 102: 409-418

Klug W, Graziani G, Grippa G, et al. 1992. Evaluation of long-range atmospheric models using environmental radioactivity data from the Chernobyl accident: ATMES Report. Amsterdam: Elsevier. 366

Lorenz E N, 1963. Deterministic non-periodic flow. J Atmos Sci, 20: 130-141

Molteni F, Palmer T N, Buizza R, et al. 1996. The ECMWF ensemble prediction system methodology and verification. Q J R Meteorol Soc, 122: $73-121$

Terada H, Katata G, Chino M, et al. 2012. Atmospheric discharge and dispersion of radionuclides during the Fukushima Dai-ichi Nuclear Power Plant accident, Part II: Verification of the source term and analysis of regional-scale atmospheric dispersion. J Environ Radioact, 112: $141-154$

Toth Z, Kalnay E. 1993. Ensemble forecasting at NMC, the generation of perturbations. Bull Amer Meteor Soc, 74: 2317-2330

Toth Z, Kalnay E. 1997. Ensemble forecasting at NCEP and the breeding method. Mon Weather Rev, 125: 3297-3319

Yamaguchi, Munehiko, Ryota S, et al. 2009. Typhoon ensemble prediction system developed at the Japan Meteorological Agency. Mon Weather Rev, 137: 2592-2604 